\title{
Estudio socioeducativo de caso mejora del clima laboral en guarderías mediante la comunicación efectiva al utilizar técnicas de programación neurolingüística
}

Socio-Educational Case Study Improving the Working Environment in Nurseries Through Effective Communication Using Neuro-Linguistic Programming Techniques

Estudo de caso socioeducativo, melhorando o ambiente de trabalho em berçários através de uma comunicação eficaz usando técnicas de programação neurolinguística

Silvina Lucia López Villagómez

Instituto Tecnológico de León, México

silvinalucia.lopez@itleon.edu.mx https://orcid.org/0000-0003-4436-2495

Claudio Rafael Vásquez Martínez

Universidad de Guadalajara, México crvasquezm@gmail.com https://orcid.org/0000-0001-6383-270X

Liliana Valdez Jiménez Universidad de Guadalajara, México dra.lilivaldez@gmail.com https://orcid.org/0000-0002-0092-2510 


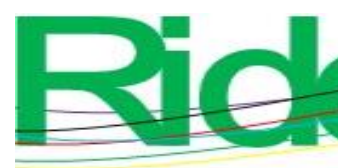

Revista Iberoamericana para la Investigación y el Desarrollo Educativo ISSN 2007-7467

\section{Abstract}

Poor communication is the cause of most of the internal problems that organizations present. When they are not resolved quickly, they can create negative feelings among colleagues, weak relationships between leaders and other employees, a bad work environment and a decrease in productivity. So, the objective of this work was to improve the working environment of two nurseries through effective communication using neuro-linguistic programming techniques. For this, a quasi-experimental design was performed, pretestposttest of a single group; in other words, an accurate assessment of the work environment was applied, areas of opportunity were identified, workshops based on neuro-linguistic programming techniques were applied, and finally the results before and after the intervention were compared. In total, 87 workers with an indefinite contract were surveyed (44 from one nursery and the rest from the other). In the previous evaluation corresponding to the first nursery, eight people of the population indicate that communication is low (18\%), 24 that it is moderate $(54 \%)$ and 12 indicate that it is optimal (28\%). In the post-intervention, 14 participants commented that it is moderate (32\%) and 30 that it is optimal $(68 \%)$, with a $p=0.005$. While in the second nursery, in the pre-intervention eight of the participants reported that communication in the organization is low (18\%), 24 moderate $(56 \%)$ and 11 optimal. (25\%) While in the post-intervention evaluation, two workers reported low communication (4\%), 12 moderate $(28 \%)$ and 29 optimal $(68 \%)(p=0.003)$. Regarding the organizational climate, in the pre-intervention phase it was found that $28 \%$ indicated that it is optimal, while in the post-intervention it rose to $57 \%$. In the second nursery, $57 \%$ of the staff in the pre-intervention indicated that the organizational climate is moderate and $25 \%$ found it optimal. In the post-intervention evaluation, the moderate rating dropped to $39 \%$ and the optimal rating increased to $61 \%$. In conclusion, it was possible to improve communication and the work environment.

Keywords: work environment, communication, neuro-linguistic programming, socioeducational. 


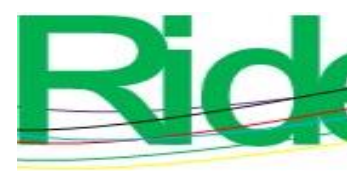

Revista Iberoamericana para la Investigación y el Desarrollo Educativo ISSN 2007-7467

\section{Resumo}

A falta de comunicação é a causa da maioria dos problemas internos que as organizações apresentam. Quando não são resolvidos rapidamente, podem criar sentimentos negativos entre colegas, relacionamentos fracos entre líderes e outros funcionários, um ambiente de trabalho ruim e uma diminuição de produtividade afetam a continuidade de cada processo que a empresa desenvolve. Portanto, o objetivo deste trabalho foi melhorar o ambiente de trabalho de dois viveiros através de uma comunicação eficaz, utilizando técnicas de programação neurolinguística. Para isso, foi realizado um desenho quase experimental, prépós-teste, de um único grupo; Em outras palavras, aplicou-se uma avaliação precisa do ambiente de trabalho, identificaram-se áreas de oportunidade, aplicaram-se oficinas baseadas em técnicas de programação neurolinguística e, finalmente, compararam-se os resultados antes e depois da intervenção. No total, foram pesquisados 87 trabalhadores com contrato por tempo indeterminado (44 de um berçário e o restante do outro). Na avaliação anterior, correspondente à creche 1 , oito pessoas da população indicam que a comunicação é baixa (18\%), 24 que é moderada (54\%) e 12 indicam que é ótima (28\%). No pós-intervenção, 14 participantes comentaram que é moderado (32\%) e 30 que é ótimo (68\%), com p =0,005. Enquanto no jardim de infância dois, na pré-intervenção, oito dos participantes relataram que a comunicação na organização é baixa (18\%), 24 moderada (56\%) e 11 ótima (25\%). Enquanto na avaliação pós-intervenção, dois trabalhadores relataram baixa comunicação (4\%), 12 moderada (28\%) e 29 ótima (68\%) ( $\mathrm{p}=0,003)$. Em relação ao clima organizacional, na fase pré-intervenção, verificou-se que $28 \%$ indicaram que é ótimo, enquanto na pósintervenção subiu para $57 \%$. Na escola maternal dois, $57 \%$ da equipe na pré-intervenção indicou que o clima organizacional é moderado e $25 \%$ o consideraram ideal. Na avaliação pós-intervenção, a classificação moderada caiu para $39 \%$ e a classificação ideal aumentou para $61 \%$. Em conclusão, foi possível melhorar a comunicação e o ambiente de trabalho.

Palavras-chave: ambiente de trabalho, comunicação, programação neurolinguística, socioeducativa.

Fecha Recepción: Febrero 2020

Fecha Aceptación: Julio 2020 


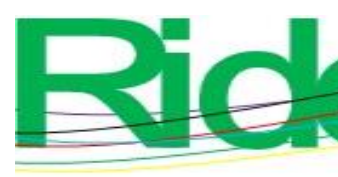

Revista Iberoamericana para la Investigación y el Desarrollo Educativo ISSN 2007-7467

\section{Introducción}

El clima organizacional es definido como el conjunto de características que distinguen a una organización de otra. Se trata de rasgos que describen a una empresa y que influyen en el comportamiento de las personas que laboran en ella (García-Solarte, 2009). "El clima organizacional comprende los efectos subjetivos, percibidos del sistema formal: estructura, responsabilidad individual, remuneración, desafío, relaciones sociales, cooperación, estándares, conflictos e identificación con la organización" (Sampieri et al, 2014, p. 50). Otros factores importantes son las actitudes, creencias, valores y motivación de las personas que trabajan en una organización (García-Solarte, 2009, p. 46).

En una investigación sobre el tema en cuestión, Tapia y Ventura (2015) encontraron que $73.68 \%$ de los colaboradores que conformaron su muestra no ponía en práctica la comunicación efectiva y $26.32 \%$ desconocía este tipo de comunicación. "Esto está afectando las relaciones interpersonales, causando daño a sus compañeros; además, la empresa al no darse cuenta de estos errores ha originado que el desempeño sea notablemente bajo" (Tapia y Ventura, 2015, p. 6).

En este sentido, si la organización no transmite a sus miembros la situación real, entonces es imposible esperar que el personal retroalimente a la organización acerca de la situación actual, es ahí donde se crea la imagen del "todo está bien" y en donde se demuestra que nadie quiere hacer nada por mejorar, porque definitivamente todo marcha bien, lo que genera resentimientos y frustraciones. La experiencia laboral en organizaciones señala la importancia de la comunicación efectiva. "Todos nos comunicamos en el trabajo. Independientemente de cuál sea nuestro campo de acción o de cuánto sepamos sobre él, el conocimiento especializado no basta para garantizar el éxito; las habilidades para comunicarnos también son relevantes" (Adler, 2005, citado en Avendaño, 2014, p. 4). Y Avendaño (2014) añade que el capital humano, es decir, las personas que integran una organización, es decisivo para el éxito de esta, ya que es el que posee las habilidades, competencias y conocimientos para el logro de los objetivos. "La buena organización y administración de este capital humano depende a su vez de la gestión de la comunicación efectiva" (Adler, 2005, p. 6). 


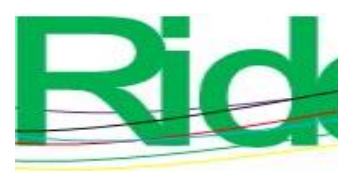

Revista Iberoamericana para la Investigación y el Desarrollo Educativo ISSN $2007-7467$

\section{Estado del conocimiento}

Los tres pilares que componen esta investigación son la programación neurolingüística, la comunicación y el clima laboral. O’Connor y Seymour (1999) definen a la programación neurolingüística como "el arte y la ciencia de la excelencia y deriva del estudio de cómo las mejores personas en distintos ámbitos obtienen sobresalientes resultados. Estas habilidades en la comunicación puede aprenderlas cualquiera para mejorar su eficiencia tanto personal como profesional" (p. 21).

El estudio del clima laboral y la comunicación efectiva son temas relevantes para toda organización, debido a que permitirá conocer la percepción que tienen los trabajadores, jefes y gerentes respecto a su entorno de trabajo y de acuerdo con su propio desenvolvimiento en la empresa. Por ello, el estudio de estas variables son necesarias, y puesto que han sido poco estudiadas, por lo tanto, esta investigación servirá como antecedente para futuros trabajos y profesionales interesados en estos temas (Tapia y Ventura, 2015). El proceso de comunicación es el que une a la organización con su entorno, así como con sus partes. Según lo menciona Ansede (2010), aproximadamente $80 \%$ del tiempo los gestores en las organizaciones lo dedican a solventar cuestiones en las que la comunicación tiene una incidencia decisiva, lo que recalca la importancia de este proceso. Además, considerando que el individuo que es capaz de comunicarse con otros de manera clara y concisa es el que logrará sus metas, las organizaciones que encuentren la manera de transmitir la información y los aspectos de la cultura que desean tener serán aquellas que lograrán sus objetivos, tal y como lo señala Naranjo (2008).

Asimismo, el adecuado manejo de las técnicas de comunicación de programación neurolingüística daría al personal y a los dirigentes de la organización las herramientas para mejorar la calidad del clima laboral; fomentaría una actitud cooperativa entre el personal, y despertaría una actitud positiva hacia su trabajo y la organización, con todo lo cual se ayudaría al crecimiento y solidificación de la empresa. 


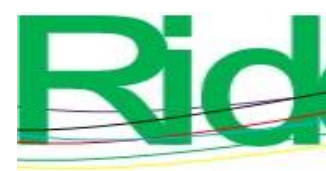

Revista Iberoamericana para la Investigación y el Desarrollo Educativo ISSN $2007-7467$

\section{Objeto de estudio}

El objeto de estudio es mejorar el clima laboral en dos guarderías mediante la comunicación afectiva, utilizando técnicas de programación neurolingüística.

\section{Objetivos}

- Analizar el clima laboral en dos guarderías mediante la comunicación afectiva, utilizando técnicas de programación neurolingüística.

- Elaborar un análisis estadístico entre las variables educación, ocupación, estado civil y las puntuaciones totales del clima laboral.

\section{Hipótesis}

Mediante la comunicación afectiva y utilizando técnicas de programación neurolingüística habrá una mejora en el clima laboral en dos guarderías.

\section{Pregunta de investigación}

¿Qué se mejora en el clima laboral de dos guarderías mediante la comunicación afectiva y utilizando técnicas de programación neurolingüística?

\section{Metodología}

En esta investigación, a un grupo se le aplicó un diagnóstico inicial para evaluar el clima laboral del personal de guardería; después se llevó a cabo la intervención, que consistió en la instrucción de técnicas de programación neurolingüística para aplicar en las organizaciones a través de la comunicación efectiva, y finalmente se realizó un diagnóstico posterior al tratamiento. Por lo que fue un diseño cuasiexperimental sin grupo control.

\section{La selección de la población}

Se tomó el total de los trabajadores 85 empleados en diferentes puestos en dos guarderías de filiales.

Como parte de los criterios de inclusión, los trabajadores realizaron una evaluación inicial y final; fueron excluidos aquellos de reciente ingreso y se descartó durante el análisis 


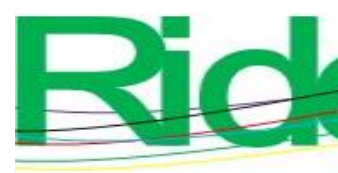

Revista Iberoamericana para la
Investigación y el Desarrollo Educativo
ISSN $2007-7467$

al personal que estuvo de vacaciones o fue separado de la institución, o que no cumplió con el proceso completo.

Las herramientas para la recolección de información fueron las siguientes:

1) Carta de consentimiento libre e informado: aquí se explica el objetivo de la investigación y que su participación consistía en contestar el cuestionario, el cual era totalmente anónimo y confidencial y cuyos datos solo se usarían para fines de esta investigación.

2) En la etapa previa a la intervención se realizó la primera evaluación del clima laboral en el personal de las guarderías para buscar áreas de oportunidad.

La escala Likert fue un diseño ex profeso para esta investigación compuesto por 56 ítems, siete áreas que conforman el clima laboral, que pueden ser influenciadas a través de la comunicación efectiva. Para cada área fueron diseñados ocho reactivos a ser evaluados, teniendo varias opciones de respuesta: se le solicitó al trabajador escoger la opción que más se acerque a la realidad que vive.

Para asegurar la validez del cuestionario de clima laboral se utilizó la técnica de validación de criterios de jueces. Por lo que una escala Likert preliminar fue sometida a la opinión de cinco profesionales dedicados al área laboral y con experiencia en el tema de elaboración de escalas. El procedimiento consistió en un análisis de contenido de los ítems con el propósito de apreciar el grado de representatividad respecto del universo y del contenido del cual forma parte. La validación lógica se realizó con base en los supuestos que guiaron la elección de los ítems; la interrogante guía a este tipo de validación fue: ¿evalúa o no este ítem la propiedad propuesta? En el caso de que fuera negativo, se cambió hasta que fue la escala aceptada en su totalidad por cada juez. Para asegurar la consistencia interna del cuestionario de clima laboral, se utilizó el cálculo del alfa de Cronbach, el cual "permite estimar la fiabilidad de un instrumento de medida a través de un conjunto de ítems que se espera que midan el mismo constructo o una única dimensión teórica de un constructo latente" (Frías, 2006, p. 3). El resultado aquí obtenido fue de 0.947, lo que quiere decir que dicho instrumento tuvo consistencia interna, según la misma Frías (2006). La escala estuvo compuesta por 56 ítems distribuidos en siete subescalas (ver tabla 1). 
Tabla 1. Encuesta del clima laboral

\section{Comunicación}

La información que recibo sobre mi trabajo es:

Cuando me dan una instrucción sé perfectamente lo que debo de hacer.

Me siento libre de expresar mis comentarios o sugerencias relacionadas con mi trabajo.

Los tableros o pizarrones de comunicación transmiten información clara e importante

Me puedo comunicar abiertamente con el personal de la institución.

En la institución existe comunicación entre los diferentes niveles.

Se me mantiene informado(a) acerca de la dirección de la compañía en general (objetivos, estrategias, desempeño, competidores, etc).

\section{Solución de problemas y conflictos}

En la institución los problemas se informan.

El personal de la institución trata de resolver los problemas con las personas involucradas.

La información circula por las vías formales más que por chismes.

El personal trata de negar los conflictos en lugar de resolverlos.

El personal trabaja en la solución de problemas.

Conozco perfectamente cuando los objetivos y metas relacionados con mi trabajo y mi departamento no se alcanzan.

Existe justicia y equidad en la solución de problemas y conflictos.

Las ideas que proporciono para mejorar el trabajo son tomadas en cuenta.

\section{Liderazgo y autoridad}

Siento que tengo una buena relación con mi jefe.

Puedo intercambiar ideas sobre diferentes aspectos de mi trabajo con mi jefe.

El ambiente de trabajo que propicia mi jefe es:
a) Muy bueno
b) Bueno
c) Malo
d) Pésimo

Mi jefe está dispuesto a ayudarme:

Mi jefe inmediato me da retroalimentación que me ayuda a mejorar mi desempeño.

Mi jefe se asegura que yo comprenda los procesos y herramientas de administración de gente incluyendo nuestras respectivas responsabilidades dentro de cada proceso. 
Tengo la oportunidad de discutir con mi jefe mis intereses de trabajo y metas en mi carrera de manera franca.

El diálogo es el medio más importante de que disponen nuestros directivos para conseguir el involucramiento de todos los empleados

\section{Cooperación y competencia intergrupal}

Las relaciones de trabajo entre mis compañeros de área son:

Me siento comprometido con mis compañeros de trabajo por lo que colaboro con ellos.

En mi área trabajamos en conjunto para lograr las metas.

Las relaciones de trabajo del personal de mi área con otras son:
a) Muy buenas
b) Buenas
c) Malas
d) Pésimas

Se siente un ambiente de trabajo cordial y cooperativo en la institución.

Las políticas de la empresa permiten que los empleados nos ayudemos mutuamente.

Dentro de mi área todos ven solo por su propio beneficio sin pensar en los demás.

Se coordinan las iniciativas de tal manera que toda el área se trabaje conjuntamente en la misma dirección.

\section{Trabajo en equipo}

Mis ideas y opiniones son valoradas y respetadas por los demás.

En mi área sabemos que los méritos del equipo son más importantes que los méritos individuales.

En mi área se valoran, agradecen y distinguen las aportaciones de los equipos y compañeros que contribuyan positivamente a los logros de la organización.

En mi departamento aprendemos de todas las experiencias sin importar si son buenas o malas.

Nos animamos unos a otros con mayor frecuencia de lo que nos criticamos.

Entre los compañeros de trabajo, nuestra comunicación se basa en la confianza, la sinceridad y el respeto mutuo.

En nuestra compañía impera una cultura de trabajo en equipo.

Aquí hay un sentido de "familia" o equipo.

\section{Actitud hacia el trabajo}

Por la mañana, me levanto con mucho ánimo para iniciar mis labores de trabajo.

Mi trabajo es monótono y aburrido. 


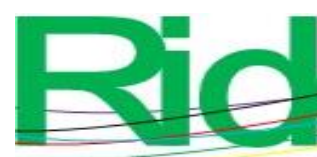

Revista Iberoamericana para la Investigación y el Desarrollo Educativo ISSN $2007-7467$

El ambiente de trabajo es pesado y frustrante.

La institución se preocupa por el respeto de la calidad de vida de sus empleados.

En general, estoy satisfecho(a) con mi institución como lugar para trabajar.

Deseo trabajar aquí por un largo tiempo.

Este es un lugar amigable para trabajar.

Estoy orgulloso de decirles a otros que trabajo aquí.

\section{Herramientas personales de comunicación}

Escucho atentamente cuando los demás hablan.

Trato de ponerme en el lugar de los otros para entenderlos.

Cuando tengo dudas, pregunto a la persona indicada.

Cuando tengo una inquietud, la comunico.

Verifico que la información que doy sea recibida de manera adecuada.

Creo que las personas son abiertas y sinceras.

Me doy cuenta cuando una persona tiene cambios de ánimo al verle sin necesidad que me platique cómo está.

Cuando hablo por teléfono identifico el estado de ánimo de mi interlocutor.

\section{Fuente: Elaboración propia}

Para la evaluación de los ítems se asignó un valor numérico a cada una de las respuestas del cuestionario. Se realizó la suma por cada área abordada y se agruparon en tres las respuestas para su análisis: bajo, moderado, óptimo.

\section{Talleres}

El contenido de los talleres se agrupó en cuatro módulos. Se diseñaron cuatro talleres de dos horas y media cada uno; en total 10 horas. Se aplicó uno cada semana durante un mes, esto para facilitar la asimilación de la información por parte de los participantes.

Los temas que se abordaron fueron: posiciones perceptuales, creencias, comunicación empática o no violenta, técnicas de negociación. 


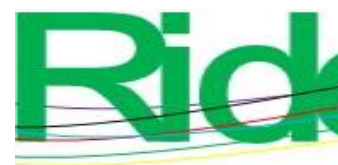

Revista Iberoamericana para la
Investigación y el Desarrollo Educativo
ISSN $2007-7467$

perciben aquellos eventos que entran en congruencia con el sistema de creencias omitiendo todo aquello que no empate con esta. El adecuado manejo del lenguaje permite impactar en el sistema de creencias; el adecuado manejo del lenguaje ayudará a lograr este objetivo. Las pautas del lenguaje indican las creencias de las personas. Por ejemplo: los conocidos como operadores modales y nominalizaciones: la persona hablará de lo que puede o no puede hacer, o debe o no debe hacer; otro ejemplo es lo que se conoce como el fenómeno de causaefecto (Dilts et al. 2013).

"Cuando usted cambia sus creencias acerca de su identidad, se convierte de algún modo en una persona distinta" (Dilts, 2013, p. 15). Por lo que es necesario mover a los individuos a un lugar diferente del que ya conocen para así generar resultados diferentes. Si lo que la persona hace no le está dando los resultados que espera debe comenzar a hacer cosas diferentes. En comunicación se habla de que el cerebro es un mecanismo cibernético, ya que se asevera que cuando la persona tiene claro el objetivo, su cerebro organizará el comportamiento inconsciente de manera que pueda alcanzarlo; por lo que la persona comenzará a realizar acciones y conductas que lo acerquen a su objetivo con la creencia de que eso es lo que debe ser (Dilts, 2004). Estas son las técnicas y conceptos que se aplican en este trabajo para lograr una comunicación eficiente dentro del ambiente organizacional para mejorar del clima laboral.

\section{Comunicación empática o no violenta}

A pesar que no es propiamente parte de programación neurolingüística, la comunicación empática o no violenta propuesta por Rosenberg (2006) es un modelo que ayuda a mejorar la comunicación con los otros a través de cuatro pasos, a saber:

a) Observación de los hechos.

b) Reconocimiento de los sentimientos de las personas involucradas.

c) Reconocimiento de las necesidades de las personas involucradas.

d) La petición concreta que se quiere hacer al otro (Rosenberg, 2006, p. 30). 


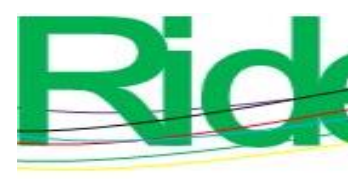

Revista Iberoamericana para la Investigación y el Desarrollo Educativo ISSN 2007 - 7467

En lo que respecta a la subescala Comunicación en la guardería uno, en la evaluación previa ocho (18\%) personas de la población señala que la comunicación es baja, 24 (54 \%) moderada y $12(28 \%)$ señala que es óptima. En la posintervención, 14 participantes se decantaron por la opción de moderada $(32 \%)$ y 30 por óptima $(68 \%)$ con una $(p=0.005)$; aquí no hubo registros de baja.

En tanto que en la guardería dos, en la preintervención ocho de los participantes reportaron que la comunicación en la organización es baja (18\%), 24 moderada (56 \%) y 11 óptima $(25 \%)$.

Mientras que en la evaluación posintervención, dos trabajadores reportaron una comunicación baja (4 \%), 12 moderada (28\%) y 20 óptima (68 \%) $(p=0.003)$.

En el área de solución de problemas, según los datos obtenidos en la evaluación preintervención de la guardería uno, 16 participantes refieren que hay un nivel bajo (36\%), 24 moderado (54\%) y 12 óptimo (28 \%); en la posintervención, cinco de los trabajadores reportaron percibir un nivel bajo 5 (11\%), 24 moderado (54\%) y 16 óptimo (36 \%). En la guardería dos, por su parte, existe también una mejora en el porcentaje de las personas que perciben esta área como óptima: pasó de 6 (14\%) a 17 (40\%).

En el tema de liderazgo, la población participante de la guardería uno lo percibe como bajo en $32 \%$ (14 trabajadores), como moderado en $48 \%$ (21 trabajadores) y como óptimo en $20 \%$ (nueve participantes). Y en la posintervención se registró como bajo con tan solo $4 \%$ (dos trabajadores), moderado con $28 \%$ (12 participantes) y óptimo con $68 \%$ (30 trabajadores) $(p=0.017)$.

Mientras que en la guardería dos, en la preintervención 15 trabajadores reportaron que el liderazgo de esta sucursal es bajo (35\%), 14 moderado (32\%) y 14 óptimo (32\%). En la posintervención cinco comentaron que es bajo (11\%), 19 moderado (44\%) y 19 óptimo $(44 \%)(p=0.03)$.

En cuestión de la cooperación y competencia intergrupal, en la guardería dos, en la evaluación preintervención, se observa que solo cinco miembros (11\%) del personal lo considera óptimo; en la evaluación posintervención esta calificación fue elegida por 23 participantes $(54 \%)(p=0.03)$. La siguiente área evaluada fue el trabajo en equipo, en la guardería uno en la evaluación preintervención se consideró bajo con 32 \%, moderado con $48 \%$ y óptimo con $20 \%$. 

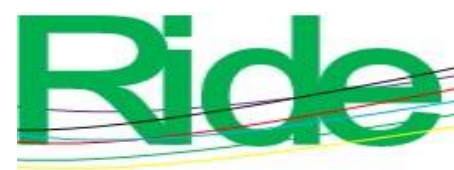

En esta área, la del trabajo en equipo, se observan también cambios en la opinión del personal de la guardería dos. Quienes lo consideraron como óptimo en la preintervención fueron tan solo 11 miembros (25\%), mientras que después de los talleres 18 participantes se decantaron por la valoración más alta (42\%).

Respecto a la actitud hacia el trabajo, en la guardería uno 14 participantes la consideraron baja (32\%), 21 moderada (48\%) y 9 óptima (20\%). Mientras que en la evaluación posintervención, seis trabajadores optaron por la opción baja (14 \%), nueve por la moderada $(21 \%)$ y 29 por óptima (66 \%). En cuestión de herramientas personales de comunicación, en la preintervención 18 miembros del personal (42\%) de la guardería dos perciben que cuentan con herramientas de este tipo; para la posintervención fueron 35 (81 \%) ( $p=0.254)$. En cuanto a la calificación del clima laboral, en la guardería uno, 20 lo consideraron bajo (46\%), 17 moderado (38\%) y siete óptimo (16\%). En tanto que en la posintervención siete optó por bajo (16\%), 23 por moderado (52\%) y 14 por óptimo (32\%) $(p=0.05)$. En este mismo rubro, los resultados de la guardería dos fueron los siguientes: ocho bajo (18 \%), 24 moderado (56 \%) y 11 óptimo (25\%). Y en la posintervención, 17 se decantó por moderado $(39 \%)$ y 26 por óptimo $(60 \%)(p=0.05)$. No hay diferencias estadísticas entre la guardería uno y dos 
Tabla 2. Análisis comparativo de dos guarderías

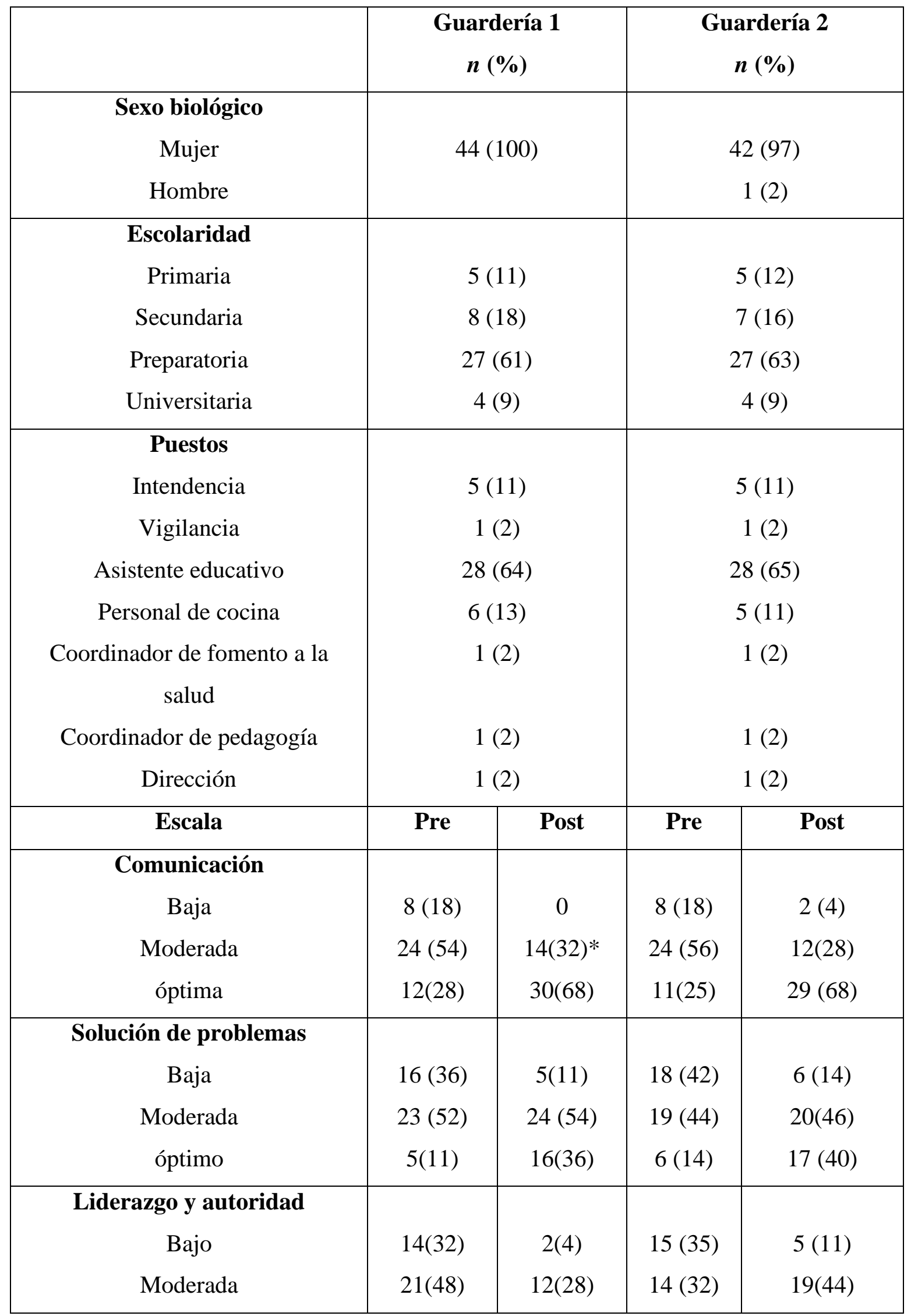


Revista Iberoamericana para la

Investigación y el Desarrollo Educativo ISSN 2007 - 7467

\begin{tabular}{|c|c|c|c|c|}
\hline Óptimo & $9(20)$ & $30(68)$ & $14(32)$ & $19(44)$ \\
\hline \multicolumn{5}{|c|}{ Cooperación y competencia } \\
\hline \multicolumn{5}{|l|}{ intergrupal } \\
\hline Bajo & $5(11)$ & $2(4)$ & $9(21)$ & $0(0)$ \\
\hline Moderado & $28(64)$ & $24(54)$ & $29(67)$ & $20(46)$ \\
\hline Óptimo & $11(25)$ & $19(43)$ & $5(11)$ & $23(54) *$ \\
\hline \multicolumn{5}{|c|}{ Trabajo en equipo } \\
\hline Bajo & $14(32)$ & $6(14)$ & $20(46)$ & $7(16)$ \\
\hline Moderado & $21(48)$ & $19(43)$ & $12(27)$ & $18(42)$ \\
\hline Óptimo & $9(20)$ & $19(43)^{*}$ & $11(25)$ & $18(42)$ \\
\hline \multicolumn{5}{|c|}{ Actitud hacia el trabajo } \\
\hline Bajo & $14(32)$ & $6(14)$ & $12(27)$ & $2(4)$ \\
\hline Moderado & $21(48)$ & $9(21)$ & $18(42)$ & 21(49) \\
\hline Óptimo & $9(20)$ & $29(66)$ & $13(30)$ & $20(46)$ \\
\hline \multicolumn{5}{|c|}{$\begin{array}{c}\text { Herramientas personales de } \\
\text { comunicación }\end{array}$} \\
\hline Bajo & $22(51)$ & $10(23)$ & $12(27)$ & $0(0)$ \\
\hline Moderado & $14(32)$ & $14(32)$ & $13(30)$ & $8(18)$ \\
\hline Óptimo & $7(16)$ & $20(45)$ & $18(42)$ & $35(81)^{*}$ \\
\hline \multicolumn{5}{|l|}{ Clima laboral } \\
\hline Bajo & $20(46)$ & 7 (16) & $8(18)$ & $0(0)$ \\
\hline Moderado & $17(38)$ & $23(52)$ & $24(56)$ & 17(39) \\
\hline Óptimo & 7 (16) & $14(32)^{*}$ & $11(25)$ & $26(60) *$ \\
\hline
\end{tabular}

$* p=0.05$ prueba de signos de Wilcoxon para pruebas relacionadas.

Fuente: Elaboración propia 


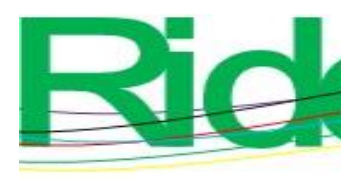
Revista Iberoamericana para la
Investigación y el Desarrollo Educativo
ISSN $2007-7467$

\section{Discusión}

Se trabajó con el supuesto de que la percepción del clima laboral del personal de las guarderías mejoraría cuando la comunicación fuera más efectiva.

Siguiendo los resultados encontrados en la guardería uno, en la evaluación preintervención se encontró que 16 \% señala que el clima organizacional es óptimo, mientras que en la posintervención subió a 32 \% la población que percibe que el clima organizacional está a este nivel.

En la guardería dos, en la evaluación preintervención se encontró que $18 \%$ del personal señala que el clima organizacional es óptimo. En la evaluación posintervención, la calificación de óptima se incrementó a $60 \%$.

Los resultados concuerdan con lo reportado en la investigación de Tapia y Ventura, (2015): al mejorar la comunicación mejora el clima organizacional. De las técnicas abordadas, algunas estuvieron basadas en los presupuestos básicos de la comunicación y los axiomas de Watzlawick et al. (1996), tal y como:

- La imposibilidad de no comunicar.

- Toda comunicación tiene un nivel de contenido y un nivel de relación.

- La naturaleza de una relación depende de la puntuación de la secuencia de comunicación entre los comunicantes.

- La comunicación humana implica dos modalidades: la digital y la analógica.

- Los intercambios comunicacionales tienden a igualar su conducta recíproca, pueden ser tanto simétricos como complementarios.

De los axiomas, se trabajaron ejercicios prácticos donde los participantes pudieron apreciar en la práctica dichos axiomas y beneficiarse de estas herramientas al entender que deben ser más conscientes de los mensajes que envían y de cómo los envían; adicional a los axiomas, se anexó el trabajo con los presupuestos puestos a continuación:

- $\quad$ La gente responde al mapa de su realidad, no a la realidad misma.

- $\quad$ Todo mapa mental es valioso.

Igualmente, los axiomas sirvieron para hacer conscientes a los participantes de los elementos de la comunicación; se buscó responsabilizarlos de los mensajes que emiten.

El trabajo de Watzlawick (1980) es uno de los pilares en el área de la comunicación que da fuerza con sus postulados al enfoque psicológico de la programación neurolingüística. 


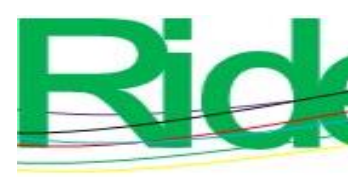

\section{Revista Iberoamericana para la Investigación y el Desarrollo Educativo ISSN 2007 - 7467}

En relación con el concepto de calibración aquí señalado, es importante recalcar que las personas lo hacen de manera empírica y en muchas ocasiones a nivel inconsciente; sin embargo, es mucho más importante que se pueda realizar esta actividad de manera consciente y adecuada para no caer en falsas interpretaciones en el lenguaje verbal o no verbal, esto fue algo que se enfatizó en los talleres. Los sistemas representacionales son una técnica que facilita la compresión de las diferentes maneras de interactuar con el mundo, mas no se debe tomar como una manera de limitar a otros. Se encontró que uno de los elementos que facilitan los problemas de comunicación es el mal hábito de no escuchar o no poner atención en el otro. Es necesario darse el tiempo y la tarea de tener una escucha activa: si solo se callara, se escuchara y se buscara llenar los huecos en la comunicación (se especificara la información) los problemas de comunicación bajarían drásticamente.

Uno de los errores de la comunicación, según Satir (1991), es la lectura de pensamiento; si se aprendiera que lo que se percibe no es la realidad, solo un simple mapa, se daría más importancia a la necesidad de la retroalimentación, y también a la manera de ejecutarla de manera efectiva para evitar herir susceptibilidades. Hacer saber al otro lo que se piensa y se quiere, si se es claro en lo que se dice y se hace saber al otro lo que se entiende, evitaría en buena proporción los malos entendidos.

Las creencias son una parte esencial en la vida. Hay creencias limitantes y creencias que se pudieran llamar potencializadoras. Las creencias limitantes tienden a encerrar en el miedo, en la imposibilidad, y si tiene en cuenta que, según Dilts et al. (1995), la mente no distingue entre ficción y realidad, las personas se dañan al estar viviendo en escenarios inapropiados que tienen solo en las mentes. Anteriormente se había hablado de que dentro de un proceso de comunicación eficiente la transmisión de información ejerce sobre el receptor el efecto deseado y que la confusión es la consecuencia de una comunicación defectuosa, donde se deja sumido al receptor en un estado de incomprensión; durante la planeación del taller, con la programación de las herramientas que se enseñarían al personal de las guarderías, se buscó que, al finalizarlo, este pudiera tener un proceso de comunicación bien logrado, y se logró avanzar en esta meta. Ahora está en manos de cada una de las personas participantes responsabilizarse de su proceso de comunicación y hacer lo que esté en sus manos para mejorarlo.

La información sobre la comunicación eficaz es el resultado de un entendimiento común entre el consumidor y el receptor (Gibson et al. 2006). Y dado que para la correcta trasmisión de la información se requieren varios aspectos, de acuerdo con Rosenberg (2006), 


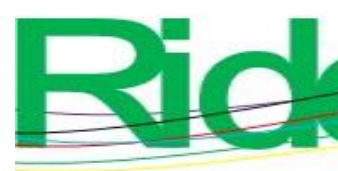

Revista Iberoamericana para la
Investigación y el Desarrollo Educativo
ISSN $2007-7467$

algunos de ellos como la comunicación verbal, la comunicación no verbal y la comunicación paralingüística, y sobre todo que exista congruencia entre ellas, es importante ayudar a las personas a poder ser más consciente de estos elementos de la comunicación y poder tener un buen manejo de su comunicación. Cuando se habla del concepto de fidelidad se puede hablar de la congruencia entre los elementos de la comunicación, es la eliminación de los componentes del ruido.

En este sentido, se dice que el clima laboral es lo que describe a una organización, la distinguen de otra, y que influye en el comportamiento de las personas que la forman, así que, aumentando el grado de mejora en la percepción del clima laboral, posiblemente hará que se gesten cambios en las conductas del personal que trabajan en las guarderías. Se plantea que el clima organizacional es la opinión que el empleado se forma de la organización, así que, una vez más, ayudando al personal a cambiar sus creencias sobre los aspectos que integran el clima laboral, se podrá mejorar la opinión que tienen de la organización. Como lo muestran los resultados obtenidos en ambas guarderías, con los talleres aumentó el grado en que las personas distinguían el clima laboral percibiéndolo como óptimo.

Según García-Solarte (2009), el clima organizacional comprende los efectos subjetivos, percibidos del sistema formal, por lo que, desde este enfoque, es viable modificar el clima laboral con técnicas de programación neurolingüística, sin embargo, es importante recordar que hay limitaciones en otros aspectos que integran el clima laboral que impactarán en su calidad, tal y como son las condiciones de trabajo y sueldos, la reciente crisis por el coronavirus que enfrenta el mundo y que ha cambiado las condiciones de trabajo.

\section{Conclusiones}

La comunicación efectiva al utilizar técnicas de programación neurolingüística puede mejorar el clima laboral. Es un modelo psicológico rápido y eficaz para lograr cambios en el capital humano. Un clima de escucha y una comunicación real conducen a la solución de problemas y con ello se mejora el clima organizacional.

La comunicación no violenta, aunque propiamente no es parte de la programación neurolingüística, es un modelo que ayuda para que se llegue a una comunicación efectiva, ya que facilita el análisis del lenguaje verbal, de las emociones y sentimientos, donde se puede, además, incluir la técnica de las posiciones perceptuales y así poder interactuar con el interlocutor de una manera más eficiente. 


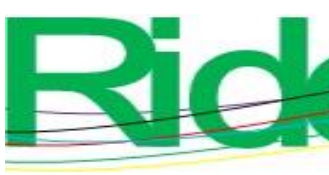

Revista Iberoamericana para la Investigación y el Desarrollo Educativo ISSN 2007 - 7467

\section{Referencias}

Adler, R. B. (2005). Comunicación organizacional. Principios y prácticas para negocios y profesiones (8. ${ }^{\mathrm{a}}$ ed.). Santa Bárbara, Estados Unidos: McGraw-Hill.

Ansede, P. (2010). La comunicación en las organizaciones en la sociedad del conocimiento. Coruña, España: Universidad de Coruña.

Asamblea Médica Mundial. (2013). Declaración de Helsinki de la Asociación Médica Mundial. Recomendaciones para guiar a los médicos en la investigación biomédica en personas. México: Conamed. Recuperado de http://www.conamed.gob.mx/prof_salud/pdf/helsinki.pdf.

Avendaño, K. (2014). La comunicación asertiva como ventaja competitiva. (ensayo). Universidad Militar Nueva Granada, Bogotá. Recuperado de https://repository.unimilitar.edu.co/handle/10654/11994.

Bandler, R. (2006). Use su cabeza para variar. Santiago, Chile: Cuatro Vientos.

Bateson, G. (1998). Pasos hacia una ecología de la mente. Buenos Aires, Argentina: LohléLumen.

Brunet, L. (2002). El clima de trabajo en las organizaciones. México: Trillas.

Dilts, R. (2004). Coaching, herramientas para el cambio. Barcelona, España: Urano.

Dilts, R. (2013). Cómo cambiar creencias con PNL. Barcelona, España: Sirio.

Dilts, R., Hallbom, T. y Smith, S. (1995). Identificación y cambio de creencias. Barcelona, España: Urano.

Dilts, R., Grinder, J., Richard, B. y De Lozier, J. (2003). Programación neuro-lingüistica Vol. 1. México: Khaos.

Frías, D. (2006). Apuntes de consistencia interna de las puntuaciones de un instrumento de medida. Universitad de Valencia, España. Recuperado de http://www.uv.es/ friasnav/AlfaCronbach.pdf.

García-Solarte, M. (2009). Clima organizacional y su diagnóstico: una aproximación conceptual. Cuaderno de Administración, (42), 43.62. Recuperado de http://www.redalyc.org/articulo.oa?id=225014900004.

Gibson, J., Ivancevich, J., Donnelly, J. y Konopaske, R. (2006). Organizaciones. México: McGraw-Hill.

Mohl, A. (2004). El aprendiz de Brujo II: El alumno magistral PNL. Barcelona, España: Sirio. 


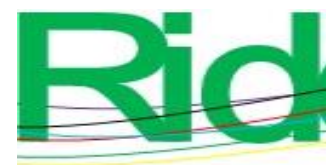

Revista Iberoamericana para la
Investigación y el Desarrollo Educativo
ISSN $2007-7467$

Naranjo, M. L. (2008). Relaciones interpersonales adecuadas mediante una comunicación y conducta asertivas. Revista Electrónica “Actualidades Investigativas en Educación”, 8(1), 1-27. Recuperado de http://www.redalyc.org/pdf/447/44780111.pdf

O’Connor, J. y Seymour, J. (1999). Introducción a la programación neurolingüística. España: Urano. Recuperado de http://www.ub.edu/hsctreballsocial/sites/default/files/pdfs/recursos/introduccion_a_1 a_pnl_p27-86_def_parte1.pdf.

Rosenberg, M. (2006). Comunicación no violenta. Barcelona, España: Urano.

Sampieri, R. H., Valencia, S. M. y Soto, R. C. (2014). Construcción de un instrumento para medir el clima organizacional en función del modelo de los valores en competencia. Contaduría y Administración, 59(1), 229-257. Recuperado de https://doi.org/10.1016/S0186-1042(14)71250-1.

Satir, V. (1991). Model: Family Therapy and Beyond. Palo Alto, United States: Science and Behavior Books.

Tapia, K. y Ventura, Y. (2015). Clima laboral y asertividad en trabajadores. (Tesis). Universidad Santo Toribio de Mogrovejo, Chiclayo. Recuperado de http://tesis.usat.edu.pe/bitstream/usat/690/1/TL_VenturaChavezYasminy_TapiaVas quezKaren.pdf.

Watzlawick, P. (1979). ¿Es real la realidad?. Barcelona: Herder.

Watzlawick, P. (1980). El lenguaje del cambio. Barcelona: Herder.

Watzlawick, P., Beavin, J. y Jackson, D. (1991). Teoría de la comunicación humana. Barcelona, España: Herder.

Watzlawick, P., Weakland, J. y Fisch, R. (1996). Cambio. Barcelona, España: Herder. 


\begin{tabular}{|c|c|}
\hline Rol de Contribución & Autor (es) \\
\hline Conceptualización & $\begin{array}{l}\text { Principal: Claudio Rafael Vásquez Martínez. IGUAL: } \\
\text { Liliana Valdéz Jiménez, Silvina Lucia López Villagómez. }\end{array}$ \\
\hline Metodología & $\begin{array}{l}\text { Principal: Silvina Lucia López Villagómez. IGUAL: } \\
\text { Claudio Rafael Vásquez Martínez, Liliana Valdéz Jiménez. }\end{array}$ \\
\hline Software & $\begin{array}{l}\text { Principal: Silvina Lucia López Villagómez. IGUAL: } \\
\text { Claudio Rafael Vásquez Martínez, Liliana Valdéz Jiménez. }\end{array}$ \\
\hline Validación & $\begin{array}{l}\text { Principal: Silvina Lucia López Villagómez. IGUAL: } \\
\text { Claudio Rafael Vásquez Martínez, Liliana Valdéz Jiménez. }\end{array}$ \\
\hline Análisis Formal & $\begin{array}{l}\text { Principal: Silvina Lucia López Villagómez. IGUAL: } \\
\text { Claudio Rafael Vásquez Martínez, Liliana Valdéz Jiménez. }\end{array}$ \\
\hline Investigación & $\begin{array}{l}\text { Principal: Silvina Lucia López Villagómez. IGUAL: } \\
\text { Claudio Rafael Vásquez Martínez, Liliana Valdéz Jiménez. }\end{array}$ \\
\hline Recursos & $\begin{array}{l}\text { Principal: Silvina Lucia López Villagómez. IGUAL: } \\
\text { Claudio Rafael Vásquez Martínez, Liliana Valdéz Jiménez. }\end{array}$ \\
\hline Curación de datos & $\begin{array}{l}\text { Principal: Liliana Valdéz Jiménez. IGUAL: Claudio Rafael } \\
\text { Vásquez Martínez, Silvina Lucia López Villagómez. }\end{array}$ \\
\hline $\begin{array}{l}\text { Escritura - } \\
\text { Preparación del } \\
\text { borrador original }\end{array}$ & $\begin{array}{l}\text { Principal: Claudio Rafael Vásquez Martínez. IGUAL: } \\
\text { Liliana Valdéz Jiménez, Silvina Lucia López Villagómez. }\end{array}$ \\
\hline $\begin{array}{l}\text { Escritura - Revisión } \\
\text { y edición }\end{array}$ & $\begin{array}{l}\text { Principal: Claudio Rafael Vásquez Martínez. IGUAL: } \\
\text { Liliana Valdéz Jiménez, Silvina Lucia López Villagómez }\end{array}$ \\
\hline Visualización & $\begin{array}{l}\text { Principal: Claudio Rafael Vásquez Martínez. IGUAL: } \\
\text { Liliana Valdéz Jiménez, Silvina Lucia López Villagómez. }\end{array}$ \\
\hline Supervisión & $\begin{array}{l}\text { Principal: Claudio Rafael Vásquez Martínez. IGUAL:, } \\
\text { Liliana Valdéz Jiménez, Silvina Lucia López Villagómez. }\end{array}$ \\
\hline $\begin{array}{l}\text { Administración de } \\
\text { Proyectos }\end{array}$ & $\begin{array}{l}\text { Principal: Liliana Valdéz Jiménez. IGUAL: Claudio Rafael } \\
\text { Vásquez Martínez, Silvina Lucia López Villagómez. }\end{array}$ \\
\hline $\begin{array}{l}\text { Adquisición de } \\
\text { fondos }\end{array}$ & $\begin{array}{l}\text { Principal: Claudio Rafael Vásquez Martínez. IGUAL: } \\
\text { Liliana Valdéz Jiménez, Silvina Lucia López Villagómez. }\end{array}$ \\
\hline
\end{tabular}

